Mens

revue d'histoire intellectuelle de l'Amérique française

MENS

\title{
La question linguistique dans la pensée de Claude Ryan au Devoir (1962-1978) : la difficile conciliation de principes nationalistes et libéraux
}

\section{Olivier Marcil}

Volume 2, numéro 2, printemps 2002

URI : https://id.erudit.org/iderudit/1024609ar

DOI : https://doi.org/10.7202/1024609ar

Aller au sommaire du numéro

Éditeur(s)

Centre de recherche en civilisation canadienne-française

ISSN

1492-8647 (imprimé)

1927-9299 (numérique)

Découvrir la revue

Citer cet article

Marcil, O. (2002). La question linguistique dans la pensée de Claude Ryan au Devoir (1962-1978) : la difficile conciliation de principes nationalistes et libéraux. Mens, 2(2), 193-231. https://doi.org/10.7202/1024609ar
Résumé de l'article

De la Commission Laurendeau-Dunton jusqu'à la loi 101, la pensée de Claude Ryan en matière de langue démontre qu'il est possible de concilier, jusqu'à un certain point, les principes libéraux et nationalistes, mais qu'en cas de conflit majeur entre les deux, le libéralisme est, chez Ryan, une valeur supérieure au nationalisme. Si le droit d'une majorité d'imposer le respect de sa langue est légitime, ce droit doit rester soumis, dans une société démocratique, aux droits reconnus aux individus et aux minorités. Si certains droits individuels doivent être subordonnés à des droits collectifs, il ne peut s'agir pour Ryan que d'une mesure exceptionnelle et ponctuelle commandée par un esprit de justice. Dans le cas du Québec, l'orientation scolaire des néo-Québécois créait un exemple de situation injuste, car elle menaçait la survie du groupe francophone. Mais l'aménagement législatif en matière de langue s'avérera de plus en plus restrictif envers les droits historiques de la minorité anglophone et les libertés individuelles au point où, avec la loi 101, il ira trop loin pour Ryan et brisera le fragile équilibre entre le respect des droits individuels et le respect des droits collectifs qu'il a tant souhaité. 


\section{LA QUESTION LINGUISTIQUE DANS LA PENSÉE DE CLAUDE RYAN AU DEVOIR (1962-1978) : LA DIFFICILE CONCILIATION DE PRINCIPES NATIONALISTES ET LIBÉRAUX ${ }^{1}$}

Olivier Marcil

\section{RÉSUMÉ}

De la Commission Laurendeau-Dunton jusqu'à la loi 101, la pensée de Claude Ryan en matière de langue démontre qu'il est possible de concilier, jusqu'un certain point, les principes libéraux et nationalistes, mais qu'en cas de conflit majeur entre les deux, le libéralisme est, chez Ryan, une valeur supérieure au nationalisme. Si le droit d'une majorité d'imposer le respect de sa langue est légitime, ce droit doit rester soumis, dans une société démocratique, aux droits reconnus aux individus et aux minorités. Si certains droits individuels doivent être subordonnés à des droits collectifs, il ne peut s'agir pour Ryan que d'une mesure exceptionnelle et ponctuelle commandée par un esprit de justice. Dans le cas du Québec, l'orientation scolaire des néoQuébécois créait un exemple de situation injuste, car elle menaçait la survie du groupe francophone. Mais l'aménagement législatif en matière de langue s'avérera de plus en plus restrictif envers les droits historiques de la minorité anglophone et les libertés individuelles au point où, avec la loi 101, il ira trop loin pour Ryan et brisera le fragile équilibre entre le respect des droits individuels et le respect des droits collectifs qu'il a tant souhaité. 


\section{ABSTRACT}

From the Laurendeau-Dunton Commission to Quebec's controversial Bill 101, Claude Ryan's attitude on linguistic issues shows that it was possible, up to a point, for him to reconcile liberal and nationalist principles. Nonetheless, when Ryan's nationalism came into conflict with his liberalism, the latter predominated. While he believed that the French-speaking majority had the legitimate right to impose the respect of its language, it also had the obligation to respect both individual and minority rights. Though Ryan was able to acknowledge that certain individual rights should be subordinated to collective rights, this could only occur in exceptional and isolated cases. In Quebec's case, the fact that most allophone immigrants traditionally opted for English-language education constituted what he believed to be an exceptional situation because it threatened the cultural and linguistic survival of the province's French-speaking majority. However, when Bill 101 began to restrict both individual rights and the English-speaking minority's historic rights, Ryan could no longer support government efforts to achieve a new linguistic equilibrium. He felt that the Parti québécois' linguistic legislation had ruptured the fragile legal and moral equilibrium be had hoped to see established between the respect of individual and collective rights.

Claude Ryan est un intellectuel dont la place dans l'histoire du Québec est certes reconnue, mais encore peu étudiée. Pourtant, Ryan s'est toujours imposé comme une figure dominante au sein d'institutions qui ont eu une influence structurante sur la société québécoise : l'Action catholique canadienne de 1945 à 1962, l'Institut canadien d'éducation des adultes de 1955 à 1961, Le Devoir de 1962 à 1978 et le Parti libéral du Québec de 1978 à 1994. De plus, son œuvre écrite et oratoire est considérable : plus de 3000 éditoriaux et articles, des centaines de conférences et discours, une dizaine de livres publiés, sans compter la pro- 
duction de programmes et de brochures du temps de l'Action catholique. Cette activité intellectuelle témoigne d'une riche pensée dont cet article vise justement à dresser un portrait évolutif. Étant donné l'espace qui nous est alloué, nous concentrerons l'analyse sur un sujet très précis, la question linguistique (et particulièrement la question de la langue d'enseignement), à une époque bien précise, la Révolution tranquille ${ }^{2}$, alors que Ryan était éditorialiste et directeur du quotidien Le Devoir .

Pour le Québec, la période 1960-1980 est une période d'aménagement linguistique sans précédent. Les différents gouvernements, autant québécois que canadien, vont successivement poser des actes et adopter des lois ayant pour objectif respectif de franciser le Québec (Commission Gendron, loi 63, loi 22, loi 101) ou de bilinguiser le Canada (Commission Laurendeau-Dunton, loi sur les langues officielles). Ces objectifs, en apparence opposés, feront l'objet de nombreux débats politiques, débats qui prendront parfois une allure émotive et violente par leur appel au sentiment nationaliste.

À la fois observateur et acteur dans ces débats, en raison du poste qu'il occupe au Devoir, Claude Ryan développe sur la question linguistique une pensée modérée, «équilibriste», qui cherche jusqu'à un certain point à concilier les objectifs de francisation du Québec et de bilinguisation du Canada, et à respecter les valeurs nationales et libérales propres à chacun. Chez Ryan, la question linguistique découle d'une prémisse nationaliste : étant donné le statut nettement inférieur au Canada du français par rapport à l'anglais, aucune égalité réelle entre les deux langues n'aura la chance d'exister sans une action gouvernementale en faveur de la langue française. Une égalité réelle des deux langues est une condition nécessaire à la recon- 
naissance officielle du dualisme (biculturalisme) canadien, c'est-à-dire de l'existence de deux nations au Canada. Par conséquent, le Québec, foyer culturel et politique des $\mathrm{Ca}$ nadiens français, devra assumer un rôle de premier plan dans la promotion du français. En revanche, cette prémisse nationaliste est rapidement balisée, dans la pensée de Ryan, par les principes supérieurs du libéralisme politique. Car si Ryan appuie les nombreuses législations linguistiques dans leur objectif de donner la priorité au français au Québec, il défendra aussi le respect des droits individuels et des droits historiques reconnus à la minorité anglo-québécoise. Ce qui nous permet de soutenir que Ryan est un libéral. Nationaliste certes, mais libéral d'abord.

\section{Du bilinguisme institutionnel au principe de territorialité}

En 1967, la Commission Laurendeau-Dunton ${ }^{4}$ dépose son Livre I sur les langues officielles dans lequel elle recommande d'enchâsser dans la constitution le français et l'anglais comme langues officielles du Canada, de proclamer le bilinguisme officiel en Ontario et au Nouveau-Brunswick et de créer des «districts bilingues», où le français et l'anglais seront utilisés dans les institutions municipales et scolaires partout où la minorité officielle constitue ou constituera dix pour cent de la population ${ }^{5}$. Au Québec, l'impact de ce rapport est immédiat, d'autant plus qu'il contient, en guise d'introduction, les "pages bleues» d'André Laurendeau, véritable cadre de référence des tenants de la théorie dualiste.

Ryan réagit favorablement à ce rapport. Il adhère avec enthousiasme au «bilinguisme institutionnel» promu par la Commission, qui signifie que les institutions canadiennes (entreprises, services gouvernementaux, hôpitaux, etc.) 
doivent offrir des services et un cadre de travail bilingues, permettant ainsi à tous les citoyens d'y travailler, de s'y épanouir et de recevoir des services dans la langue officielle de leur choix ${ }^{6}$. Ce programme fait appel, écrit-il, à «une conception humaniste et épanouissante de l'égalité» et à une «forme supérieure de vie civilisée ${ }^{7}$ ».

Au Québec, toutefois, l'accueil réservé au bilinguisme institutionnel est mitigé. Si, pour certains, il s'agit de la reconnaissance bienvenue d'une revendication séculaire, pour d'autres, c'est trop peu, trop tard : il devient un obstacle à la volonté de franciser le Québec ${ }^{8}$. Chez les fédéralistes nationaliste ${ }^{9}$ on affirme que l'on pourrait concilier cette volonté de francisation avec un bilinguisme institutionnalisé si l'on accordait une place satisfaisante au principe de territorialité, selon lequel le bilinguisme ou l'unilinguisme s'appliquera en fonction de la composition linguistique du territoire.

La légitimité du principe de territorialité fut vivement discutée au sein de la Commission Laurendeau-Dunton ${ }^{10}$. Laurendeau estimait que la survie d'une langue minoritaire en milieu bilingue dépendait de l'existence de régions unilingues. Par conséquent, il souhaitait proclamer unilingues françaises certaines régions du Québec. Mais la majorité des commissaires - Frank Scott en tête - se sont opposés à ce principe parce qu'ils le jugeaient incompatible avec la conception égalitaire soutenue par le bilinguisme institutionnalisé. Ils ont par contre accepté que certaines mesures institutionnelles tiennent compte des particularismes territoriaux : dans le Livre I, l'institutionnalisation du bilinguisme pour seulement deux provinces, l'Ontario et le Nouveau-Brunswick, et la proclamation de «districts bilingues» là où la minorité officielle constitue au moins dix pour cent de la population; dans le Livre III, la création, à 
l'intérieur de chaque institution fédérale, «d'unités de travail francophones» et la recommandation de faire du français la principale langue de travail dans le secteur privé au Québec. Le compromis, fragile, permettait de sauvegarder l'essentiel du bilinguisme institutionnalisé. Au Québec cependant, le principe de territorialité l'emportera. Le gouvernement québécois en fera la pierre d'assise de sa propre politique linguistique. Une politique qui, de l'avis de plusieurs (et en particulier de Scott et de Pierre Trudeau), viendra miner l'esprit du bilinguisme institutionnalisé et la pertinence de la loi sur les langues officielles.

Adoptée en 1969, la loi fédérale sur les langues officielles est la première consécration des recommandations de la Commission Laurendeau-Dunton. Pour Ryan, l'entreprise est "capitale», car elle instaure le bilinguisme institutionnalisé au Canada, mais elle est aussi "partielle» et "préliminaire ${ }^{11} »$. Préliminaire, car s'il s'agit d'un aspect nécessaire du bilinguisme officiel au Canada, ce n'est qu'un premier pas vers une solution au vrai problème canadien : la reconnaissance du biculturalisme canadien.

Si le bilinguisme est un problème réel, dit Ryan, il ne saurait être substitué au problème fondamental de la crise canadienne, soit l'égalité des deux sociétés, peuples ou nations qui constituent le Canada. Cet aspect de la pensée de Ryan est fondamental. Il constitue la prémisse nationaliste de sa réfléxion : la langue étant un fondement de la nation, le bilinguisme est indissociable du biculturalisme (ou binationalisme).

Le bilinguisme n'est que le visage officiel et extérieur d'une réalité qu'il faut appeler, faute d'un meilleur terme, le biculturalisme ${ }^{12}$.

Une langue, si elle est vivante et surtout si elle est parlée par un nombre suffisant de personnes, 
n'existe pas dans l'air pur. Elle donne naissance à une communauté, laquelle tend à s'enraciner de préférence dans un territoire donné, puis à se doter d'institutions originales, lesquelles forment, quand elles sont suffisamment développées, une société (ou nation) distincte ${ }^{13}$.

Il n'y a pas seulement deux langues et deux cultures principales au Canada. Il y a d'abord et surtout deux communautés, deux sociétés, deux nations. Aussi longtemps qu'on ne voudra pas reconnaître plus clairement cette réalité, on s'exposera à gaspiller comme cela s'est fait depuis 7 ans - des millions de dollars en programmes de saupoudrage linguistique dont les résultats demeureront limités ${ }^{14}$.

Le Canada anglais doit [...] savoir que jamais le Québec, tant que durera le déséquilibre actuel entre les deux «nations», ne consentira à troquer une once de sa liberté pour une montagne de bilinguisme dans le reste du Canada ${ }^{15}$.

C'est sur cette prémisse nationaliste qu'il appuiera les politiques de francisation au Québec et ce, malgré leurs objectifs parfois contradictoires avec la politique de bilinguisation qu'il avait entérinée. C'est aussi que le contexte politique change à la fin de la décennie 1960 : Pierre Trudeau devient Premier ministre, le gouvernement du Canada devient plus centralisateur et homogénéisateur et il démontre une ferme volonté de promouvoir le multiculturalisme aux dépens du biculturalisme. D'un autre côté, la loi sur les langues officielles connaît des ratés et atteint ses limites (le bilinguisme institutionnel ne peut s'étendre à la langue d'enseignement, de travail, d'affichage ou d'administration provinciale ou municipale sans la participation des autorités provinciales et municipales) ${ }^{16}$. L'incapacité d'enrayer le déclin démographique de la popula- 
tion francophone hors Québec, l'érosion du français même au Québec et l'intégration massive des immigrants à la communauté anglophone font comprendre à Ryan l'ampleur de l'utopie qui sous-tend le bilinguisme institutionnel pancanadien ${ }^{17}$. Dans ce contexte, il prend définitivement conscience de la nécessité pour le Québec de changer luimême les règles du jeu en matière de politique linguistique s'il veut assurer la survie d'une nation francophone au Canada.

La Commission Gendron ${ }^{18}$ marque le début d'un rapatriement de l'initiative linguistique au Québec. Elle fut créée en décembre 1968 par le gouvernement de l'Union nationale, dans la foulée des troubles linguistiques qui surviennent à Saint-Léonard à chaque rentrée scolaire depuis quelques années. Elle a le mandat de recommander des mesures propres à assurer le plein épanouissement et la diffusion de la langue française au Québec dans tous les secteurs d'activités (éducation, culture, économie, etc.), en s'attaquant prioritairement aux questions du français comme langue de travail, de l'intégration des néo-Québécois à la communauté francophone du Québec et des droits linguistiques de la minorité anglophone ${ }^{19}$. Désormais, au Québec, l'objectif de donner la priorité au français l'emporte sur le principe de l'égalité des deux langues.

Déjà, quelques mois avant la mise sur pied de la Commission Gendron, Ryan préconisait un changement d'orientation dans la politique linguistique québécoise. Il affirmait avec force que l'État québécois devait prendre ses responsabilités en matière de langue. Le gouvernement du Québec, disait-il, a une «responsabilité historique spéciale» envers la langue et la culture de la majorité de ses citoyens, il doit donc assurer la priorité du français sur le territoire du Québec $^{20}$. «Aucune égalité réelle n'a de chance d'exister [en- 
tre la langue française et la langue anglaise au Canada], écritil, si une priorité raisonnable n'est accordée au français dans la politique linguistique du gouvernement québécois ${ }^{21} . »$ Cette opinion sera le pilier de sa pensée en matière de langue pour les années à venir.

Mais comment expliquer une telle position chez un homme que nous qualifions de libéral? Le gouvernement du Québec ne devrait-il pas souscrire, au nom de la liberté, à l'égalité des deux langues officielles sur son territoire? Normalement oui, dit Ryan. La langue française, parlée par $80 \%$ des Québécois, devrait être laissée au dynamisme de la vie et le gouvernement du Québec ne devrait pas avoir à intervenir en faveur des droits linguistiques d'une collectivité majoritaire. Mais la réalité du Québec est différente. Le Québec est la seule société nord-américaine où l'on parle français. Il est un îlot perdu dans un océan anglophone. Il subit d'énormes pressions de la part de la culture et de la langue anglaise à travers la télévision, la radio; les revues. Il existe donc un déséquilibre entre les deux langues que seule peut rétablir une action «raisonnable» - Ryan insiste sur le mot - de l'État québécois en faveur du français ${ }^{22}$.

Une fois établie cette nécessité, il reste à la concilier avec les exigences d'égalité qu'avaient établies la Commission Laurendeau-Dunton et auxquelles, rappelons-le, Ryan avait adhéré : le rejet de la race et du groupe ethnique au profit de la communauté linguistique comme fondement de la politique de la langue ${ }^{23}$, la reconnaissance juridique de l'égalité des deux langues officielles dans les services publics, et la liberté de chaque citoyen d'appartenir, pour des fins officielles, à la communauté linguistique de son choix. Ces trois principes sont les frontières qu'on ne peut franchir sans nier le principe d'égalité des deux langues, et à 
l'intérieur desquelles on doit penser les moyens pour favoriser le français, soutient Ryan ${ }^{24}$.

Telle est sa position en 1968. Cependant, nous verrons qu'il évoluera, au début des années 1970, vers une position plus coercitive et qu'il finira, lors du débat sur la langue d'enseignement, par remettre implicitement en question le troisième postulat. En attendant, les initiatives visant à accorder une priorité au français proposées par le directeur du Devoir sont de type incitatif (mettre en place une politique d'information et d'incitation en faveur de l'école française auprès des néo-Canadiens, exiger des écoles anglaises un enseignement beaucoup plus poussé du français, favoriser dans la vie économique un meilleur usage du français, etc.). La voie de l'incitation sera aussi empruntée par le rapport Gendron, déposé à la fin de décembre 1972. Les recommandations Gendron sont modérées, voire hésitantes. Ainsi, la recommandation la plus marquante invite à faire du français la seule langue officielle du Québec tout en proclamant l'anglais et le français «langues nationales ${ }^{25}$ ».

Entre 1968 et 1972, l'opinion de Ryan évolue rapidement. Il comprend désormais qu'il est impossible d'assurer la prépondérance de la langue française au Québec sans l'application de certaines mesures coercitives. Par conséquent, il aurait préféré que la Commission Gendron recommande des mesures plus fermes, mieux définies ${ }^{26}$. Qui plus est, c'est à ce moment qu'il glisse définitivement vers le principe de territorialité (sans toutefois abandonner l'ensemble des implications du bilinguisme institutionnel). Un bilinguisme authentique et réel est désormais un bilinguisme où coexistent deux langues fortes et dynamiques, où «une vaste unité de langue française fai $[t]$ pendant à une vaste unité de langue anglaise ${ }^{27} \gg$ sans que l'une puisse nuire à l'épanouisse- 
ment de l'autre. Une telle perspective lui apparaît désormais plus réaliste.

On constate une fois de plus, en examinant les données du recensement de 1971, la fragilité d'une politique qui ferait reposer nos principaux espoirs sur l'avènement illusoire du bilinguisme à travers tout le pays. [...] Le Canada est destiné de toute évidence à demeurer un pays anglophone. Le Québec a, pour sa part, d'excellentes chances de rester un pays francophone [...] Entre les deux sociétés, suivant les formes que l'avenir réserve aux structures politiques, il devra s'instituer des formes plus ou moins élaborées de bilinguisme institutionnel. Ces structures resteront en quelque sorte marginales par rapport à la vie réelle, quoique nécessaires et très utiles. $\mathrm{Ce}$ n'est pas de ce côté que nous devons investir nos principaux espoirs politiques ${ }^{28}$.

Ce constat aura toutefois son revers conséquent : la priorité accordée à l'anglais dans les autres provinces canadiennes devient légitime.

Ni l'Alberta, ni même l'Ontario ne se sont senties tenues, à la suite de l'adoption de la loi fédérale sur les langues officielles, d'imposer sur leur territoire les mêmes normes linguistiques que définit la loi Trudeau (langues officielles). Chacune a continué au contraire de vivre et de fonctionner en donnant une immense priorité à la langue anglaise dans les actes de son administration publique. [C'est] une ligne de conduite qui obéit à un sain réalisme ${ }^{29}$.

On ne peut assister à une application plus concrète de la théorie dualiste. D'ailleurs, Pierre Trudeau y verra une négation de la fonction intégrative du bilinguisme : «Le bilinguisme unit les citoyens; le dualisme les divise. Le bilinguisme signifie que vous pouvez parler aux autres; le 
dualisme signifie que vous pouvez vivre dans une langue et le reste du Canada dans une autre ${ }^{30} \%$. Le fossé entre deux visions du Canada - entre deux nationalismes, diront certains - se creuse.

\section{Les droits linguistiques des minorités : droits fondamentaux ou droits historiques?}

Malgré les implications de sa position sur le statut prioritaire de la langue française au Québec, nous sommes d'avis que Claude Ryan est un libéral. Le droit individuel est fondamental et premier chez lui, même si son libéralisme accorde une certaine importance aux droits collectifs. Des droits collectifs par contre qui n'existent pas en eux-mêmes, mais qui doivent être un "prolongement normal» des libertés individuelles. "Aucun groupe, quel qu'il soit, écrit Ryan, n'a le droit d'ériger son propre bien, au mépris des libertés personnelles et d'une conception du bien général embrassant à titre égal tous les citoyens ${ }^{31}{ }^{\prime} »$. Si l'État a un rôle à jouer pour assurer certains droits collectifs, il est hors de question qu'il se mette au service exclusif d'un groupe de citoyens, aussi majoritaire soit-il.

Les droits des minorités sont, pour moi, antérieurs à ceux de l'État. Ils le sont tellement que dans ma pensée, aucun groupe majoritaire n'a le droit de s'approprier les rouages de l'État pour imposer sa culture aux autres ${ }^{32}$.

[...] la reconnaissance des droits des minorités relève non pas d'abord du bon plaisir des États ou de l'intérêt des groupes majoritaires, mais de la justice et de considérations morales fondées sur une certaine conception de l'homme ${ }^{33}$.

Toutefois, dans la pensée de Ryan - et nous en verrons un bel exemple avec ses écrits sur la langue d'enseigne- 
ment -, les droits et les libertés individuelles n'ont pas une valeur absolue si elles ne sont pas synonymes de justice. En d'autres mots, l'idée de justice permet chez Ryan de limiter temporairement l'exercice de certains droits individuels (c'est d'ailleurs le principe qui est à la base du système judiciaire dans les États démocratiques). S'il était prouvé que certaines conditions d'inégalité socio-économiques tendent à favoriser indûment la minorité au détriment de la majorité et, par conséquent, créent une situation d'injustice, l'État pourrait établir des mesures spéciales et temporaires en faveur de la majorité afin de rétablir l'égalité ${ }^{34}$.

Au Québec, les relations entre la communauté anglophone et la communauté francophone furent marquées par un particularisme historique peu commun : le maintien d'une majorité faible dans une condition d'infériorité par une minorité forte. Or, depuis la Révolution tranquille, la majorité francophone a pris conscience politiquement d'elle-même ${ }^{35}$ et s'affirme de plus en plus dans un néonationalisme que Ryan considère positif et $\operatorname{sain}^{36}$. S'il est évident que la société québécoise doit travailler à instaurer un équilibre linguistique plus fidèle à sa réalité démographique, Ryan soutient en retour qu'une politique linguistique favorable au français ne devra pas nier à la communauté anglophone les droits que lui confèrent tant le "droit natureb» (droits fondamentaux de l'homme) que l'histoire (droits qui ne sont pas fondamentaux, mais qui sont tout de même reconnus par la législation) ${ }^{37}$. Car, en raison de sa conception dualiste du Canada, Ryan considère que la communauté anglo-québécoise doit bénéficier d'un statut spécial au sein du Québec. «Le cas canadien-français constitue, à l'intérieur de l'ensemble canadien, un cas unique et distinct. Il en va de même pour le cas des anglophones à l'intérieur de l'ensemble québécois ${ }^{38}$." 
Le conflit scolaire de Saint-Léonard a sans doute été l'événement catalyseur qui a forcé le gouvernement du Québec à s'aventurer dans l'explosif dossier linguistique. Rappelons que c'est la chute du taux de natalité des francophones du Québec et le choix massif par les immigrants de l'école anglaise plutôt que française qui est à la base du conflit. Ce choix se traduit par l'assimilation des immigrants au groupe anglophone et, par conséquent, il constitue une menace à la survie du groupe francophone au Québec. La crise éclate en 1967 alors que les commissaires d'école de Saint-Léonard décident de remplacer les classes dites «bilingues» (où les trois quarts des cours sont en fait dispensés en anglais) par des classes unilingues françaises. Les ItaloQuébécois, en majorité anglophones, font alors valoir leur droit au libre choix de la langue d'enseignement devant les tribunaux et le gouvernement. L'imbroglio prend rapidement une envergure nationale et violente. En 1969, les manifestations tournent à l'émeute.

Pour Ryan, la chute du taux de natalité des francophones et l'assimilation massive des immigrants à la communauté anglophone laisse entrevoir de sombres perspectives de survie pour la communauté francophone, des perspectives devant lesquelles les francophones ne peuvent rester indifférents : «Un peuple qui demeurerait indifférent en face de ces perspectives serait un peuple indigne de vivre. Il ne mériterait plus de conserver sa place dans la famille des nations $\mathrm{s}^{39}$.» Une intervention gouvernementale destinée à corriger cette situation est donc justifiée ici. Par contre, Ryan précise qu'on ne saurait non plus nier le droit à la croissance, à l'épanouissement d'une minorité reconnue en transférant, par voie législative, sa capacité d'assimilation vers le groupe majoritaire. Pris dans ce dilemme, il va re- 
chercher une solution qui sera à la fois respectueuse des valeurs nationales et des valeurs libérales.

Par conséquent, il rejette la solution de l'unilinguisme. Cette solution est inapplicable pour une société pluraliste qui appartient à l'Amérique du Nord, dit-il. Elle procure l'effet pervers de camper encore plus la communauté anglophone dans son isolement plutôt que de lui offrir la possibilité de participer pleinement à la construction du Québec moderne. Qui plus est, il s'agit d'une mesure qui nie les droits séculaires de la communauté anglophone.

[...] en niant un droit personnel au profit d'un droit collectif, [la majorité] oublie que le culte du droit collectif ne peut que conduire à un dangereux totalitarisme s'il n'est pas compris comme le prolongement, et non comme la négation d'un droit personnel. Le droit personnel, en cette matière, c'est le droit des parents, proclamé par la déclaration des droits de l'homme en 1948, de choisir par priorité le genre d'éducation à donner à leurs enfants. Le droit collectif, dans son acception la plus large, c'est le droit d'une société ou d'une communauté humaine de se doter d'un système d'enseignement apte à réaliser les objectifs qu'elle s'est fixés. [...] On peut, au nom de la justice, exiger de l'État qu'il prenne des dispositions spéciales en vue d'aider, de soutenir positivement une communauté culturelle assiégée dans sa vie même. On ne saurait toutefois, sans injustice, exiger qu'au nom de l'intérêt de cette communauté, l'État efface d'un trait de plume des droits d'autres communautés vivant dans son sein. [...] Là où le droit personnel s'exprime sous des formes suffisamment diverses pour que cela constitue un fait social d'importance, la loi du second, c'est celle du pluralisme et non de l'uniformitét ${ }^{40}$. 
Cet extrait le démontre bien : Ryan reconnaît le droit de la collectivité francophone d'agir politiquement sur la situation linguistique, mais ce droit ne peut nier ni le droit individuel ni les droits historiques de la minorité anglophone. Toutefois, cet extrait contient aussi une ambiguité : quand Ryan écrit que «le droit personnel, en cette matière, c'est le droit des parents, proclamé par la déclaration des droits de l'homme en 1948, de choisir par priorité le genre d'éducation à donner à leurs enfants», est-ce que cela signifie qu'il considère le droit des Anglo-Québécois de posséder leur propre système d'éducation et le principe du libre choix de la langue d'enseignement comme des droits fondamentaux? Est-ce que le droit personnel est ici synonyme de droit fondamental ou de droit naturel? L'extrait ci-dessus peut laisser croire que oui. Mais à peine deux mois plus tard, en mars 1969, s'il écrit de nouveau que le droit des parents au choix de la langue d'enseignement est le corollaire indispensable de la reconnaissance de droits linguistiques à la minorité anglophone, il prend soin d'ajouter que ce droit n'a pas de valeur absolue :

Le droit des parents [...] est, à nos yeux, un corollaire du droit plus large des minorités culturelles et linguistiques. Il se présente comme le premier, l'indispensable corollaire d'une reconnaissance des droits linguistiques d'une minorité. Il n'a certes pas, de ce fait, la valeur absolue et aveuglément contraignante que certains porte-parole anglophones ont évoquée ${ }^{41}$.

Autrement dit, le droit qui en janvier 1969 pouvait sembler un droit fondamental est en fait un droit histori$q^{4} e^{42}$. Cette nuance est capitale pour la suite de notre démonstration parce qu'une personne qui, à l'instar de Ryan, est libérale ne peut pas accepter que l'on porte atteinte aux droits fondamentaux. En revanche, elle peut toujours ac- 
cepter, sans sortir du cadre libéral, de réduire la portée de certains droits historiques reconnus à des minorités, surtout s'ils sont injustifiés ou discriminatoires ${ }^{43}$.

À la suite de l'affrontement violent de septembre 1969 à Saint-Léonard, le gouvernement unioniste de Jean-Jacques Bertrand décide finalement d'intervenir et accouche en moins d'un mois du bill $63^{44}$. Dans la foulée des débats entourant le projet de loi, la position de Ryan sur les droits historiques des anglophones évolue rapidement. Premièrement, il contredit ce qu'il affirmait en janvier 1969 sur la reconnaissance internationale des droits scolaires des minorités :

Quand M. [François-Albert] Angers affirme qu'on a tort de se réclamer des grands principes du droit international pour défendre les droits scolaires des anglophones, il a raison. On ne saurait l'accuser d'étroitesse sans faire en même temps le procès d'à peu près tous les gouvernements du monde et de la communauté internationale ${ }^{45}$.

Ce qui confirme que, dans sa pensée, les droits scolaires des minorités relèvent du droit historique et non du droit fondamental $^{46}$.

Deuxièmement, après avoir favorisé l'usage de mesures incitatives pour assurer la primauté du français, il change d'opinion et reconnaît qu'il est désormais nécessaire d'user de contraintes particulières et immédiates, mais des contraintes modérées, intelligentes et sélectives, pour s'attaquer au problème d'assimilation des immigrants à la communauté anglophone. Il propose d'obliger tous les résidents du Québec qui sont en attente d'obtention de leur citoyenneté canadienne à inscrire leurs enfants à l'école française. Une fois l'immigrant devenu un citoyen à part entière, il 
pourra exercer, comme tous les autres citoyens, sa liberté de choix en matière d'enseignement.

Nous avons longtemps cru que l'incitation devrait suffire. Notre opinion a changé sur ce point précis. Nous croyons maintenant que, durant la durée de la période de probation, tous les non-Canadiens qui s'établissent à demeure au Québec devraient être tenus d'inscrire leurs enfants à l'école française ${ }^{47}$.

Certains accuseront Ryan d'instituer ainsi une discrimination entre les citoyens et les non-citoyens. Il répliquera que la contrainte est minime et loin d'être antilibérale, car elle se compare aux autres cas de limites posées à l'exercice des privilèges ou des droits qui sont réservés aux citoyens canadiens (le droit de vote, le droit d'accéder à des charges publiques électives, etc.).

Le gouvernement Bertrand ne retiendra pas la proposition du directeur du Devoir et va plutôt proclamer, par la loi 63, le libre choix de la langue d'enseignement, tout en décrétant des mesures incitatives pour favoriser l'intégration des immigrants à l'école française. Ryan aurait préféré que le gouvernement «fit montre de plus de réalisme, qu'il tînt davantage compte de conditions nouvelles créées par l'histoire et qu'il nuançât en conséquence sa conception de la libertét8\%.

\section{Le critère le moins injuste : la langue maternelle}

En 1974, convaincu de l'inefficacité de la loi 63 et fort des recommandations du rapport Gendron, le gouvernement libéral de Robert Bourassa décide d'aller de l'avant avec une politique linguistique globale : la loi 22. Entretemps, la liberté de choix réservée aux citoyens canadiens, que le directeur du Devoir avait vivement défendue lors du débat entourant le bill 63, s'est à nouveau restreinte devant 
la poursuite de l'assimilation massive des immigrants par le groupe anglophone. Désormais, Ryan va défendre avec vigueur le critère de la langue maternelle (la première langue apprise et encore comprise) comme déterminant de l'inscription des enfants des immigrants à l'école française ou anglaise au Québec (une solution aussi préconisée par le Conseil supérieur de l'Éducation du Québec). Il écrit, et il est intéressant de constater l'évolution avec sa position originelle :

Le corollaire normal d'une politique de priorité du français, c'est que soient appelés à fréquenter l'école française tous les enfants qui ne se rattachent pas, par leur culture et leur langue maternelle, au groupe minoritaire qui jouit d'un traitement d'exception. Cela s'étant toujours pratiqué au Québec sans qu'une obligation légale pèse à cette fin sur les francophones, nous ne voyons pas pourquoi on créerait à leur intention une contrainte spéciale, à moins que l'expérience récente n'en démontre la nécessité. Dans le cas des immigrants, au contraire, l'expérience du dernier siècle a fait voir la puissance d'attraction démesurée du contexte nord-américain. Il faut mettre un terme à la saignée qui se produit de ce côté depuis la fin du dernier conflit mondial ${ }^{49}$.

Alors qu'en mars 1969, il affirmait que le corollaire indispensable de la reconnaissance des droits de la minorité linguistique était le droit des parents (sans exception) de choisir la langue d'enseignement de leurs enfants, Ryan circonscrit désormais, chez les immigrants, cette liberté de choix aux seuls parents qui se rattachent au groupe anglophone "par leur culture et leur langue maternelle». À son avis, il n'y a aucune obligation d'étendre à tous les immigrants «un traitement d'exception» réservé à une minorité qui, en vertu de l'histoire et de la configuration du pays 
canadien, n'est pas une minorité comme les autres. Le critère de la langue maternelle permettra à la minorité anglophone de continuer à s'accroître par l'apport des immigrants en provenance de pays de langue et de culture anglaises, tout en assurant aux francophones que les enfants des immigrants non-anglophones seront inscrits à l'école française. Quant aux citoyens qui résident déjà au Québec, peu importe leur langue maternelle, ils ne seraient pas privés des droits dont ils jouissent déja ${ }^{50}$. L'obligation faite aux immigrants ne serait pas indéfinie. Jusqu'à la onzième année d'enseignement, plaide Ryan ${ }^{51}$. Après, les immigrants auront les mêmes droits que tous les autres citoyens.

Cette position institue une distinction entre les immigrants anglophones (qui auront les mêmes droits que les citoyens) et les immigrants non-anglophones. Ryan le reconnait et regrette que sa position soit discriminatoire, mais comparativement au libre choix, celle-ci lui apparaît la moins dommageable et la moins injuste.

Que l'on établisse en ce faisant une distinction, nul ne le niera. Mais la vie en établit depuis vingt-cinq ans une autre qui parait encore plus injuste parce qu'elle attaque l'avenir même de la communauté francophone. Le choix devant se faire entre deux voies imparfaites, il faut opter pour celle qui, sans créer d'injustice, offre les meilleures garanties de paix et d'équilibre pour l'avenir ${ }^{52}$.

Libéral, il cherchera néanmoins à atténuer au maximum l'effet de cette discrimination. Il va donc, quelques mois plus tard, demander aux citoyens francophones de restreindre eux aussi leurs droits individuels en s'interdisant l'inscription de leurs enfants à l'école anglaise. Pour lui, c'est une question de responsabilité, de devoir, mais surtout une question de justice. Après tout, c'est en leur 
nom que les droits individuels des immigrants non-anglophones sont restreints. La distinction ne se fera plus entre deux classes d'immigrants, mais selon la langue parlée, citoyens actuels et futurs citoyens confondus ${ }^{53}$. Une position qui illustre bien la réconciliation dans sa pensée des valeurs libérales et des valeurs nationales.

Pourtant, le critère de la langue maternelle constitue un accroc aux postulats de la Commission LaurendeauDunton. Rappelons que Ryan avait spécifié, en 1968, qu'il rejetterait toute politique linguistique qui contredirait implicitement ou explicitement les postulats du rapport Laurendeau-Dunton, balises obligées, selon lui, d'une politique de francisation respectueuse de l'égalité des deux langues. Or sa pensée a évolué ici car sa position sur la langue d'enseignement va à l'encontre du troisième postulat : la liberté de chaque citoyen d'appartenir, pour des fins officielles, à la communauté linguistique de son choix. En effet, par l'utilisation du critère de la langue maternelle, on obligerait tous les parents non-anglophones à inscrire leurs enfants à l'école française au Québec. Ainsi, sur le territoire québécois, le gouvernement forcerait, au moyen de l'éducation, l'intégration des immigrants non-anglophones à la communauté linguistique francophone et il interdirait aux francophones d'intégrer leurs enfants à la communauté anglophone. Au Québec donc, seuls les citoyens et les immigrants dont la langue maternelle est l'anglais resteraient totalement libres de choisir leur communauté linguistique d'appartenance. Les autres perdraient ce droit pour une période temporaire, soit jusqu'à ce que leurs enfants terminent le secondaire.

Aux prises avec le même dilemme que Ryan, le gouvernement Bourassa cherche une formule qui gardera intact le principe de liberté de choix dans la langue d'ensei- 
gnement, tout en restreignant la capacité assimilatrice du groupe anglophone. Avec la loi 22, il tranche en faveur d'une épreuve d'aptitude linguistique pour tous les enfants que l'on souhaite inscrire à l'école anglaise. Le but de l'épreuve est d'établir si l'enfant possède une «connaissance suffisante» de la langue anglaise pour lui permettre d'être inscrit dans une école anglophone. Advenant un échec à l'examen, l'enfant devra obligatoirement être inscrit à l'école française. Cette épreuve sera ouverte sans exception à tous les enfants, ce qui, de l'avis du gouvernement, préserve le principe du libre choix. La loi prévoit aussi d'imposer des quotas d'élèves anglophones par district scolaire. Ces quotas ne pourront être augmentés ou encore, une école anglaise ne pourra être construite dans un district, sans la présence d'un nombre suffisant d'élèves de langue anglaise pour l'alimenter.

Sur la langue d'enseignement, Ryan est en désaccord avec la loi 22 qu'il considère "nébuleuse», "ambiguë», «arbitraire» et «laxiste» ${ }^{54}$. Non seulement la notion de «connaissance suffisante» de la langue anglaise comme fondement des tests linguistiques est laissée au bon jugement du ministre de l'Éducation, ce qui ouvre carrément la porte au pouvoir discrétionnaire du ministre et à tous les problèmes d'application qui en découleront, mais surtout, on s'expose à faire proliférer les initiatives qui visent à faire apprendre l'anglais aux enfants d'immigrants et de francophones par des cours intensifs et privés afin qu'ils acquièrent la «connaissance suffisante» pour passer avec succès l'examen du ministre. Avec un tel projet de loi, le gouvernement rate la cible, dit Ryan, parce que les tests ne limitent pas réellement le pouvoir assimilateur de la minorité anglophone et qu'ils permettent aux immigrants de prendre des initiatives pour contourner la loi ${ }^{55}$. Qui plus est, Ryan décrit les 
quotas d'élèves anglophones par district scolaire comme une initiative carrément antilibérale parce qu'elle institue une discrimination en fonction du lieu de résidence et non de la langue. Selon que les parents demeureront dans un district où les écoles anglaises auront fait le plein de leur capacité d'accueil, selon qu'ils demeureront dans un district où se produit un développement domiciliaire important, ils seront astreints en matière de choix scolaire à un régime différent de celui des autres parents ${ }^{56}$. Ce qui restreint pour ces parents le droit au libre choix que le gouvernement proclamait d'entrée de jeu pour tous.

La solution Bourassa est imparfaite. D'ailleurs, plusieurs estimeront qu'elle sera une cause importante de sa défaite électorale du 15 novembre 1976. Elle démontre surtout à quel point il est difficile d'intervenir en faveur d'un groupe sans accepter de faire quelques entorses aux principes libéraux. Certes, la solution préconisée par Ryan restreint un droit individuel (le droit reconnu par la Déclaration des droits de l'homme de 1948 de choisir, par priorité, le genre d'éducation à donner à ses enfants), elle institue une certaine discrimination selon la langue maternelle (d'un côté, les francophones et les allophones, de l'autre, les anglophones) et elle sort des balises d'égalité linguistique fixées par la Commission Laurendeau-Dunton. Mais c'est le prix à payer, le moins injuste selon lui, pour assurer la survie de la communauté linguistique francophone. À son avis, on peut difficilement trouver un équilibre plus raisonnable entre les droits individuels et les droits collectifs. Par conséquent, il refusera d'aller plus loin dans l'imposition de mesures coercitives. Dans sa pensée, les limites permises au sein desquelles les principes libéraux peuvent être restreints viennent d'être atteintes. 
Une minorité bafouée : les Anglo-Québécois et la loi 101

Alors que la loi 22 a restreint l'école anglaise à ceux qui savaient prouver qu'ils maîtrisaient la langue anglaise, le projet de Charte de la langue française (projet de loi 1), déposé en 1977 par le gouvernement péquiste de René Lévesque, va plus loin encore en ne le permettant qu'à ceux dont l'un des parents a reçu l'enseignement primaire en anglais au Québec. Il s'agit donc d'une application beaucoup plus contraignante du critère de la langue maternelle que ne l'entendait Ryan. En fait, le gouvernement Lévesque reconnaît les droits acquis de la minorité anglophone du Québec en matière d'enseignement, mais il refuse, à la différence de Ryan, de faire une distinction (qu'il juge discriminatoire) entre les immigrants anglophones et les autres. Bref, les droits reconnus ne prévaudront que pour les anglophones déjà installés au Québec.

La position péquiste relève d'une conception de la place de la minorité anglophone au Québec qui est à l'opposé de celle de Ryan. De l'avis de Camille Laurin, ministre titulaire de la Charte de la langue française :

La nation québécoise est composée, non pas d'une majorité francophone et d'une minorité anglophone, mais bien d'une majorité francophone et de plusieurs minorités de langues différentes. Face à la langue officielle, toutes les minorités doivent être traitées sur un pied d'égalité. [...] Du fait du caractère historique de la minorité anglaise, il convient qu'elle conserve un réseau d'institutions scolaires. [...] L'usage de l'anglais, langue internationale, est admis chaque fois que la nécessité en est démontrée et, comme langue d'une minorité, de la même manière que les autres langues des autres minorités ${ }^{57}$. 
Défenseur d'une vision dualiste du pays canadien, Ryan est choqué par cette diminution, voire cette négation du «statut particulien» de la minorité anglophone du Québec :

La réalité concrète de Montréal, en tout cas, c'est qu'il y a deux communautés linguistiques possédant chacune une vie propre. À côté de celles-là, les autres communautés ne sont pas négligeables, loin de là. Mais prétendre ramener la communauté anglophone à l'une de celles-ci, c'est prendre ses rêves pour la réalité et poser le germe de coûteuses injustices ${ }^{58}$.

Qu'on le veuille ou non, les anglophones forment en effet au Québec une communauté nettement identifiée; ils y occupent une place historique dont les racines remontent à plus de deux siècles; ils sont au surplus assez nombreux pour avoir droit à une reconnaissance raisonnable de leurs droits linguistiques et culturels. Or, rien dans le projet de loi $\mathrm{n}^{\circ} 1$ ne laisserait soupçonner cette réalitée $e^{59}$.

La position de Laurin s'inspire d'une dynamique exprimée dans la plupart des pays unitaires, et particulièrement en France : la reconnaissance officielle d'un seul État, d'une seule nation et d'une seule langue officielle ${ }^{60}$. Selon cette conception de la démocratie allergique aux particularismes, les droits linguistiques des Anglo-Québécois sont des privilèges historiques discriminatoires envers les autres minorités. Dans une société qui se veut moderne et démocratique, les citoyens doivent tous être traités de la même façon face à l'État, peu importe leur origine ethnique. Il est donc impératif d'abolir toutes les formes de privilèges et de rétablir l'égalité de tous les citoyens devant la loi. Un argument séduisant qui sera aussi utilisé par les partisans de la déconfessionnalisation des écoles publiques, puisqu'il défendent une conception démocratique similaire. Mais pour 
Ryan, le projet de Charte de la langue française, loin d'être démocratique, relève plutôt d'un nationalisme «étroit et chauvin ${ }^{61} »$ et constitue, par la réduction considérable des droits (ce ne sont pas des privilèges) de la minorité angloquébécoise, une rupture avec la théorie dualiste. À cet égard, dit-il, la loi 22, malgré ses imperfections, était beaucoup plus près de ses conceptions de la vie, du droit et de la justice $^{62}$.

Ryan est d'accord avec les indépendantistes quand ils affirment que la minorité anglo-québécoise a toujours bénéficié du traitement le plus libéral en Amérique du Nord. Mais selon lui, le Québec doit préserver ce traitement libéral et être fier de l'exemple de civisme et de démocratie qu'il donne à toutes les communautés pluralistes du monde. Si l'on reconnait des droits historiques aux deux grandes familles linguistiques, ces droits doivent s'étendre aux membres de chaque famille linguistique, indépendamment de leur date de naissance ou de leur implantation au Québec, réaffirme-t-il. Voilà une politique plus libérale. La politique du gouvernement Lévesque, qui réserverait l'accès à l'école anglaise aux seuls enfants dont les familles anglophones sont déjà installées au Québec et y ont reçu leur éducation, va complètement à l'encontre de ce principe. On institue une distinction entre citoyens natifs du Québec et les futurs citoyens et il est discriminatoire d'interdire aux parents anglophones d'envoyer leurs enfants à l'école anglaise à cause du lieu où ils sont nés, ou encore, parce qu'ils auraient fait l'effort dans leur jeunesse de fréquenter l'école française. Pis encore, cette mesure entraînerait à long terme l'extinction de la communauté anglo-québécoise si celle-ci s'avère incapable d'assurer sa croissance par le seul jeu des naissances ${ }^{63}$. 
À la lumière de ce qui précède, peut-on affirmer que Ryan démontre une fois de plus qu'il est un libéral? Nous croyons que oui. Le critère de la langue maternelle avec restriction pour les francophones, malgré certains accrocs aux droits individuels, est plus près d'une conception libérale de la démocratie que la position de Laurin. Alors que Ryan cherche à assurer les droits collectifs de la communauté francophone par le moins de coercition possible sur les droits individuels, Laurin ne semble pas en faire autant, au contraire. Qui plus est, le projet de loi 1 souhaite instituer la supériorité des droits collectifs sur les droits individuels en décrétant la primauté de la Charte de la langue française sur la Charte québécoise des droits de la personne de 1975, pourtant un document beaucoup plus fondamen$\mathrm{tal}^{64}$.

Pour qui se veut libéral, la primauté doit obligatoirement revenir au droit individuel. Ce qui, manifestement, est l'avis de Ryan :

Dans une société démocratique, la charte des droits de la personne doit venir en premier lieu. Une charte des droits linguistiques, si elle est nécessaire, doit s'inscrire en prolongement du premier document ou à tout le moins s'y intégrer harmonieusement sans que soient sacrifiés des droits fondamentaux garantis par celui-ci ${ }^{65}$.

Le gouvernement Lévesque se trouve à utiliser abusivement le pouvoir de l'État pour satisfaire le groupe majoritaire francophone, soutient-il. Le problème est d'autant plus grave que la Charte de la langue française entre à plusieurs reprises en opposition avec la Charte québécoise des droits et libertés, notamment sur la définition du "peuple québécois». Dans le préambule du projet de loi 1 , il est écrit que : 
L'Assemblée nationale constate que la langue française est, depuis toujours, la langue du peuple québécois et que c'est elle qui lui permet d'exprimer son identité. L'Assemblée nationale reconnaît la volonté des Québécois d'assurer la qualité et le rayonnement de la langue française [...] L'Assemblée nationale entend poursuivre cet objectif dans un climat de justice et d'ouverture à l'égard des minorités qui participent au développement du Québec ${ }^{66}$.

Ryan constate que si cette définition du peuple québécois trouve certains fondements dans l'histoire et la réalité concrète du Québec, elle pose, par sa formulation, d'importantes conséquences juridiques pour les Québécois non francophones. Il voit à travers cette façon de définir le peuple québécois, une confusion entre l'appartenance à un groupe culturel majoritaire et l'appartenance à la société politique et, par conséquent, une contradiction avec le principe d'égalité de tous les citoyens devant la loi sans égard pour l'origine ethnique, qui est à la base de la démocratie libérale. Pris littéralement, déplore Ryan, le préambule laisse entendre qu'il y aurait d'une part les Québécois, c'est-àdire les francophones qui sont ici "depuis toujours», et d'autre part, les minorités qui ne font que "participer au développement du Québec» et dont le monde se situe en marge du monde francophone, en marge du monde "québécois». Ryan exigera que l'on élargisse la définition de peuple québécois et que l'on interprète celle-ci exclusivement dans son sens politique et libéral, c'est-à-dire de façon à ce qu'elle «embrasse sur un pied d'égalité tous les citoyens du Québec. [...] Il faut dire tout simplement que tous les citoyens qui résident au Québec et y paient des taxes sont des Québécois à part entière ${ }^{67} \gg$. Aussi, ajoute-t-il, le gouvernement Lévesque doit assurer que les droits à l'égalité 
actuellement reconnus par la Charte québécoise des droits de la personne ne seront aucunement «annihilés ou diminués» par la législation linguistique ${ }^{68}$.

En résumé, le projet de loi 1 est celui qui, parmi les législations linguistiques instituées ou envisagées successivement par les différents gouvernements du Québec pendant les décennies 1960 et 1970, accorde la plus grande place - une place démesurée pour Ryan - aux droits collectifs de la majorité francophone. À travers ses éditoriaux, il tente de ramener le gouvernement péquiste à une solution plus centriste, plus respectueuse des droits individuels. Afin de faciliter la compréhension de sa pensée sur la question, nous avons procédé à une schématisation des solutions législatives en matière de langue d'enseignement envisagées depuis la fin des années soixante (schéma ci-dessous). Les solutions sont classées graduellement, selon qu'elles accordent une priorité aux droits individuels ou aux droits collectifs.

\section{Législations possibles en matière de langue d'enseignement}

\section{Primauté des droits individuels}

1) Retour au libre choix de la langue d'enseignement (bill 63);

2) Maintien de la loi 22 (et des tests d'aptitude linguistique);

3) Solution du Conseil supérieur de l'Éducation (accès à l'école anglaise pour tous les enfants de langue maternelle anglaise, sans égard à leur lieu d'origine);

4) "Clause Canada» (accès à l'école anglaise pour tous les enfants natifs du Québec ou non dont l'un des parents a fréquenté l'école élémentaire anglaise au Canada);

5) Solution du projet de loi 1 ou "Clause Québec» (accès à lécole anglaise pour tous les enfants natifs du Québec dont l'un des parents a fréquenté l'école élémentaire anglaise au Québec).

Primauté des droits collectifs 
D'emblée, Ryan rejette les solutions extrémistes (numéros 1 et 5). Si le libre choix est la solution la plus attrayante en théorie libérale, elle aboutira à des résultats inacceptables en pratique. En revanche, la solution du projet de loi 1 est assurément celle qui produirait les résultats les plus avantageux pour la communauté francophone, mais elle est fondée sur une conception nationaliste discriminatoire des droits de la minorité anglophone.

La loi 22 (numéro 2) est une mesure à abandonner car elle tend, par le biais de tests, à favoriser l'accès à l'école anglaise de nombreux élèves qui devraient normalement se diriger vers l'école française.

La solution du Conseil supérieur de l'Éducation (numéro 3) s'avère la meilleure, répète Ryan. Elle reconnait la place historique de la communauté anglophone du Québec, évite toute discrimination fondée sur le lieu d'origine et accorde à la communauté anglophone la possibilité de se renouveler. Elle se situe à mi-chemin (en équilibre, dirait Ryan) entre les droits individuels et les droits collectifs.

Enfin, à défaut de la solution du Conseil supérieur de l'Éducation, la "clause Canada» (numéro 4) pourrait constituer un compromis acceptable, écrit Ryan, tout en précisant qu'il s'agit du «seuil minimal en deçà duquel il serait irréaliste et injuste d'aller ${ }^{69} \%$. Il est intéressant de mentionner que ce "seuil minimal» est celui qui sera consacré en 1982 par l'article 23 de la Charte canadienne des droits et libertés.

Mais en août 1977, avec la loi 101, le gouvernement Lévesque optera pour la "clause Québec» (numéro 5). Acerbe, Ryan écrira qu'il s'agit d'un «recul dangereux et stupide» qui reflète bien le nationalisme "pessimiste, étroit et chauvin» du gouvernement Lévesque ${ }^{70}$. Il ira même jusqu'à demander l'intervention de la Cour suprême du Ca- 
nada afin de faire respecter le droit de tout Canadien, francophone ou anglophone, de réclamer que ses enfants soient instruits dans leur langue maternelle dans toute province, là où un nombre minimum d'enfants le justifie (clause $\mathrm{Ca}$ nada). Un droit qui devrait être constitutionnalisé par le biais d'une charte canadienne des droits afin qu'il soit respecté par tous les gouvernements provinciaux, ajoute-t-il ${ }^{71}$. En réalité toutefois, l'opinion de Ryan est relativement isolée au sein des francophones du Québec, en majorité favorables à la charte ${ }^{72}$.

Impuissant devant la résurgence d'un nationalisme qu'il juge obscurantiste et réducteur, on voit se dessiner le désir de l'intellectuel de se muer en homme d'action. Le saut en politique devient de plus en plus évident. C'est donc sans surprise que le 10 janvier 1978, Ryan annonce sa démission du journal qu'il dirige depuis 1964 pour se joindre au Parti libéral du Québec. Un parti politique qui, de son propre avis, se reconnait dans les thèmes qu'il défend depuis quinze ans : primauté des libertés individuelles et défense des intérêts du Québec. Parmi les priorités qu'il se fixe alors se trouve la restauration de l'équilibre entre l'affirmation collective des francophones et le respect des droits des minorités ${ }^{73}$.

\section{$* * *$}

La pensée de Claude Ryan sur la question linguistique offre un bel exemple de conciliation des valeurs libérales et nationales. Après s'être explicitement prononcé en faveur du bilinguisme institutionnel et de l'égalité des langues française et anglaise d'un océan à l'autre, Ryan prend conscience du danger que cette approche laisse planer sur la collectivité francophone en Amérique du Nord et décide de favoriser une approche plus territoriale de la ques- 
tion linguistique. Répondant à cette prémisse nationaliste, il sera d'avis qu'aucune égalité réelle entre les deux langues n'aura de chance d'exister sans accorder un statut prioritaire à la langue française sur le territoire du Québec. Conséquemment, à travers les différentes initiatives linguistiques, sa position a évolué en faveur de la reconnaissance de droits linguistiques pour les francophones du Québec. Mais, parce qu'il est foncièrement libéral, il a tenté en même temps d'encadrer ces droits collectifs afin de restreindre au maximum toute discrimination dont pourraient être victimes les néo-Québécois et les Anglo-Québécois. Son opposition à la loi 101 démontra, en définitive, que malgré son attachement à certaines valeurs nationales, Ryan est d'abord un libéral car il refusa de soumettre les libertés individuelles fondamentales aux valeurs nationales.

\section{NOTES}

${ }^{1}$ Cet article fait partie d'un ouvrage à paraître prochainement aux Éditions Varia sur la pensée politique de Claude Ryan à l'époque où il était directeur du Devoir. Remerciements au professeur Pierre Trépanier pour ses conseils et son soutien.

${ }^{2}$ Nous considérons ici la Révolution tranquille dans son sens large, c'est-à-dire la période allant de la victoire libérale de juin 1960 au référendum de mai 1980, une période caractérisée par le développement continu de l'État-providence québécois, le déclin accéléré de l’Église catholique et la croissance rapide d'un nouveau nationalisme francoquébécois.

${ }^{3}$ Claude Ryan fit son entrée au Devoir en juin 1962 comme éditorialiste. En 1964, suite au départ de Gérard Filion, il en deviendra le directeur et il le restera jusqu'en janvier 1978, alors qu'il démissionna pour devenir le chef du Parti libéral du Québec.

${ }^{4}$ Commission royale d'enquête sur le bilinguisme et le biculturalisme co-présidée par André Laurendeau et Davidson Dunton. 
${ }^{5}$ Recommandations reproduites dans Jean-Claude Corbeil, L'aménagement linguistique du Québec, Montréal, Guérin, 1980, p. 43-47.

${ }^{6}$ Commission royale d'enquête sur le bilinguisme et le biculturalisme, Livre I, Les langues officielles, Ottawa, Imprimeur de la Reine, 1967, p. XVIII. Le bilinguisme institutionnel se distingue du bilinguisme individuel, qui est plutôt l'obligation pour les individus d'apprendre une seconde langue. Ryan approuve cette volonté de travailler à un bilinguisme institutionnel plutôt qu'individuel, mais il évite de rejeter la nécessité du bilinguisme individuel, particulièrement pour les francophones qui doivent vivre dans une réalité socio-économique nord-américaine majoritairement anglophone. Le défi du continent invite à une combinaison des deux types de bilinguismes, soutient-il. Claude Ryan, "Pour sortir d'un dilemme tragique», Le Devoir, 19 décembre 1969.

${ }^{7}$ Claude Ryan, «Le rapport Laurendeau-Dunton : une invitation à une forme supérieure de vie civilisée», Le Devoir, 7 décembre 1967.

${ }^{8}$ Paul-André Linteau, René Durocher, Jean-Claude Robert et François Ricard, Histoire du Québec contemporain, Tome II, Le Québec depuis 1930, nouvelle édition révisée, Montréal, Boréal Compact, 1989, p. 601.

${ }^{9}$ Cette expression est empruntée à Jean-H. Guay et François Rocher («De la difficile reconnaissance de la spécificité québécoise», dans François Rocher, dir., Bilan québécois du fédéralisme canadien, Montréal, VLB, 1992, p. 58-78) qui l'utilisent pour identifier les nationalistes québécois qui revendiquent des changements constitutionnels en faveur du Québec tout en s'opposant à l'option souverainiste.

${ }^{10}$ Voir à ce sujet Léon Dion, La révolution déroutée 1960-1976, Montréal, Boréal, 1998, p. 211, 214.

${ }^{11}$ Claude Ryan, «La première pierre d'un édifice complexe», Le Devoir, 9 juillet 1969.

${ }^{12}$ Claude Ryan, «Bilinguisme ou biculturalisme?», Le Devoir, 11 novembre 1963. Nous soulignons.

${ }^{13}$ Claude Ryan, «Deux communautés linguistiques ou deux sociétés?», Le Devoir, 27 janvier 1968.

${ }^{14}$ Claude Ryan, «Le débat sur le bilinguisme à Ottawa», Le Devoir, $1^{\text {er }}$ juin 1973. Nous soulignons. 
${ }^{15}$ Claude Ryan, «Le bill C-120 : une pièce maitresse d'un programme de bilinguisme pour le Canada», Le Devoir, 21 mai 1969.

${ }^{16}$ Corbeil, op. cit., p. 47-48; Linteau et al., op. cit., p. 601.

${ }^{17}$ Claude Ryan, "Où en est "l'utopie" du bilinguisme», Le Devoir, 28 novembre 1974.

${ }^{18}$ Commission d'enquête sur la situation de la langue française et sur les droits linguistiques au Québec présidée par le linguiste Jean-Denis Gendron.

${ }^{19}$ Corbeil, op. cit., p. 49.

${ }^{20}$ Claude Ryan, «Une politique linguistique pour le Québec. 2- Pas d'égalité vraie sans une priorité raisonnable du français», Le Devoir, 6 septembre 1968.

${ }^{21}$ Ibid.

${ }^{22}$ Ibid.

${ }^{23}$ Les mots race et groupe ethnique doivent être entendus ici selon le sens exclusif qu'a voulu leur donner la Commission, c'est-à-dire une référence identitaire au sens biologique ou héréditaire du terme (droit du sang). Commission royale d'enquête sur le bilinguisme et le biculturalisme, op. cit., p. XII-XV.

${ }^{24}$ Claude Ryan, «Une politique linguistique pour le Québec. 1- Les normes sociologiques et juridiques», Le Devoir, 5 septembre 1968.

${ }^{25} \mathrm{La}$ volonté des commissaires était en fait d'établir des dispositions particulières à la langue anglaise en raison de la place spéciale qu'occupe la communauté anglophone au Québec, ce que Ryan approuvera fortement. Mais il déplorera le choix du terme «nationab» pour qualifier la langue anglaise, ce qui, affirme-t-il, «ne pouvait que donner lieu, dans le contexte québécois, à de regrettables confusions». En effet, il est pour le moins délicat d'identifier la langue anglaise comme la langue «nationale» d'une collectivité qui se définit de plus en plus comme une nation d'expression française. Voir Claude Ryan, "Trois niveaux majeurs d'intervention», Le Devoir, 13 novembre 1973.

${ }^{26}$ Claude Ryan, «Le rapport Gendron (1)», Le Devoir, 19 février 1973.

${ }^{27}$ Claude Ryan, «Les hauts et les bas du bilinguisme», Le Devoir, 25 mars 1974. 
${ }^{28}$ Claude Ryan, «Quelques leçons du dernier recensement», Le Devoir, 27 avril 1972. Nous soulignons.

${ }^{29}$ Claude Ryan, "L'unité canadienne : des écarts dangereux», Le Devoir, 31 janvier 1976. Nous soulignons.

${ }^{30}$ Pierre Elliott Trudeau (avec la collaboration de Ron Graham), Trudeau, l'essentiel de sa pensée politique, Montréal, Le Jour, 1998, p. 148.

${ }^{31}$ Claude Ryan, «Libertés individuelles et libertés collectives», Le Devoir, 13 décembre 1967.

${ }^{32}$ Claude Ryan, «Le Québec idéal selon le RIN», Le Devoir, 12 mai 1966.

${ }^{33}$ Claude Ryan, «Un seul critère?», Le Devoir, 3 mars 1969.

${ }^{34}$ Claude Ryan, "Quelques implication de l'unilinguisme. 2- La formule des professeurs de français du Québec», Le Devoir, 24 janvier 1969.

${ }^{35}$ En fait, cette prise de conscience avait déjà émergé durant les années trente, mais elle fut en quelque sorte trahie par l'Union nationale. Les années soixante marquent le renouveau de son affirmation et son triomphe.

${ }^{36}$ Claude Ryan, "La communauté anglophone dans le Québec d'aujourd'hui», Le Devoir, 12 février 1966.

${ }^{37}$ Ibid.

${ }^{38}$ Ryan, loc. cit., 3 mars 1969.

${ }^{39}$ Claude Ryan, «St-Léonard, problème politique» Le Devoir, 4 septembre 1968.

${ }^{40}$ Claude Ryan, "Quelques implication de l'unilinguisme. 1- La formule des professeurs de l'école normale Laval», Le Devoir, 23 janvier 1969.

${ }^{41}$ Ryan, loc. cit., 3 mars 1969.

${ }^{42}$ Tout comme le droit de posséder son propre système d'éducation d'ailleurs. En effet, lors des manifestations du mouvement «McGill français», en mars 1969, Ryan va défendre la légitimité du réseau scolaire anglophone au nom des droits historiques de la minorité angloquébécoise. Aurélien Leclerc, Claude Ryan, Québec, Mémoire de M.A. (Sciences politiques), Université Laval, 1977, p. 43. 
${ }^{43}$ C'est d'ailleurs ce qu'a défendu le politologue de l'Université Laval, François Blais. Selon ce dernier, le libéralisme permet l'expression du nationalisme tant que ce dernier ne contrevient pas aux droits moraux fondamentaux ou prioritaires (c'est-à-dire le droit de propriété, les libertés relatives à l'intégrité de la personne, la liberté de pensée et de conscience, les libertés politiques). Selon Blais, les autres droits sont des droits secondaires qui n'ont pas la même importance morale au sein du cadre libéral et qui, par conséquent, ne sont pas inaliénables. Voir François Blais, «Peut-on être libéral et nationaliste?», dans François Blais, Guy Laforest et Diane Lamoureux, dir., Libéralisme et nationalismes, Sainte-Foy, PUL, 1995, p. 19-32.

${ }^{44}$ Projet de loi 63. Le mot «bill» fut, jusqu'aux années soixante-dix, le mot anglais couramment utilisé dans les procédures parlementaires au Québec pour désigner une loi. Fait révélateur de la situation linguistique de l'époque : on appelait «bill» un projet de loi qui avait pour objectif de faire la promotion de la langue française.

${ }^{45}$ Claude Ryan, «Dans le feu de l'action», Le Devoir, 31 octobre 1969.

${ }^{46}$ En entrevue, Ryan nous a confirmé que même s'ils sont très importants, les droits linguistiques (et pas seulement les droits scolaires) ne sont pas, selon lui, des droits fondamentaux au même titre que la liberté d'expression ou la liberté de pensée, par exemple.

${ }^{47}$ Cité dans Leclerc, op.cit, p. 80.

48 Ibid., p. 92.

${ }^{49}$ Claude Ryan, «Ce que pourrait être une solution réaliste», Le Devoir, 26 février 1974.

\section{${ }^{50}$ Ibid.}

${ }^{51}$ Claude Ryan, «La loi d'abord, les règlements ensuite», Le Devoir, 14 mai 1974.

${ }^{52}$ Ryan, loc. cit., 26 février 1974.

${ }^{53}$ Ryan, loc. cit., 14 mai 1974. Dans le même ordre d'idée, Ryan ne demande pas de restreindre le libre choix de la langue d'enseignement pour les anglophones. Ainsi, les transferts linguistiques ne peuvent se faire qu'à sens unique, en faveur de la communauté francophone.

${ }^{54}$ Dans ses autres aspects (travail, administration publique, etc.), il considère que la loi 22 atteint un équilibre fort satisfaisant. 
${ }^{55}$ Claude Ryan, «La solution la plus nette», Le Devoir, 15 mai 1974; «Le projet de loi 22 et la langue d'enseignement, Le Devoir, 7 juin 1974; «M. Cloutier serait-il seul à avoir le pas?», Le Devoir; 15 juillet 1974; «L'indispensable minimum», Le Devoir, 16 juillet 1974.

${ }^{56}$ Claude Ryan, «Deux logiques difficilement superposables», Le Devoir, 17 juillet 1974.

${ }^{57}$ Camille Laurin, Le français, langue du Québec, Montréal, Éditions du Jour, 1977, cité dans Corbeil, op. cit., p. 98-99.

${ }^{58}$ Claude Ryan, «Des chiffres tronqués, des exemples mal choisis» dans Vers une charte de la langue franfaise au Québec: Recueil de textes sur le Livre blanc et le projet de loi $n^{\circ}$ 1. Montréal, 1977 p. 50. (Coll. "Les dossiers du Devoin)).

${ }^{59}$ Claude Ryan, «Le caucus libéral et le projet de loi n ${ }^{\circ}$ ", Le Devoir, 12 juillet 1977.

${ }^{60}$ Ce qu'Angéline Martel appelle un (modèle stato-nationab», un modèle homogénéisateur dont deux principes fondateurs sont la territorialisation (souveraineté) et l'unification par le droit, un modèle qui s'apparente à «l'État nationab» de Ryan. Voir Angéline Martel, «La politique linguistique canadienne et québécoise. Entre stratégie de pouvoir et identités», Globe. Revue internationale d'études québécoises, vol. $2, \mathrm{n}^{\circ} 2,1999$, p. $38-42$.

${ }^{61}$ Claude Ryan, «La porte étroite du Docteur Laurin» dans Une société stable, Montréal, Héritage, 1978, p. 194.

${ }^{62}$ Claude Ryan, «Le livre blanc sur la langue» dans Vers une charte..., op. cit., p. 77.

${ }^{63}$ Ibid., p. $94-95$

${ }^{64}$ Claude Ryan, «Un dangereux carcan», dans Vers une charte..., op. cit., p. 92-93.

${ }^{65}$ Claude Ryan, «Politique linguistique et droits humains», dans Vers une charte..., op. cit., p. 101.

${ }^{66}$ Ibid., p. 23-24.

${ }^{67}$ Claude Ryan, «Pour un définition généreuse de Québécois», dans Une société stable..., op. cit., p. 207. 
${ }^{68}$ Ryan parle ici de l'article 10 (aucune personne ne doit être victime de discrimination fondée sur la langue), de l'article 28 (toute personne en état d'arrestation a le droit d'être informée dans sa langue) et de l'article 43 (les personnes appartenant à des minorités ethniques ont le droit de maintenir et de faire progresser leur propre vie culturelle avec les autres membres de leur groupe) de la Charte québécoise des droits de la personne. Ces droits sont des droits à l'égalité de traitement et non des droits linguistiques au sens strict, soutient-il. Par conséquent, ils ne doivent pas être diminués par la Charte de la langue française. Ibid., p. 208.

${ }^{69}$ Claude Ryan, «La Charte du français sous la loupe du droit», Le Devoir, 5 juin 1977.

${ }^{70}$ Claude Ryan, «La guillotine à Québec», Le Devoir, 23 octobre 1977.

${ }^{71}$ Claude Ryan, "Pourquoi dire non tout de suite?», Le Devoir, 23 juillet 1977. À première vue, cette opinion émise par un nationaliste québécois qui a toujours demandé des modifications dans le mode de nomination des juges de la Cour suprême, a de quoi surprendre. Ryan laisserait-il à une cour dont les magistrats sont exclusivement nommés par le gouvernement fédéral le soin d'interpréter une charte qui pourrait remettre en question certains aspects capitaux de l'aménagement linguistique québécois? Les textes analysés ne le disent pas, mais nous pouvons en douter. La constitutionnalisation, dans une charte canadienne des droits, du droit de tout Canadien, francophone ou anglophone, de réclamer que ses enfants soient instruits dans leur langue maternelle dans toute province, là où un nombre minimum d'enfants le justifie sera reprise en 1980 dans le Livre beige du Parti libéral du Québec, parti politique dont Ryan est devenu le nouveau chef en 1978. Or, le Livre beige prendra soin de réclamer d'ambitieuses modifications dans la nomination des juges et dans le fonctionnement de la Cour suprême. Voir Commission constitutionnelle du Parti libéral du Québec, Pour une nouvelle fédération canadienne, Montréal, Parti libéral du Québec, 1980, p. 31-34, 59-62.

${ }^{72}$ Lucia Ferretti fait remarquer que, depuis 1956, le Devoir a toujours traduit fidèlement la réflexion de la société québécoise sur elle-même, l'opinion publique et ses choix politiques à l'exception du débat autour de la loi 101, "comme si, à cette occasion, le journal avait perdu contact avec une opinion francophone généralement favorable à la charte». Voir Lucia Ferretti, "Idées et structures, 1964-1993», dans Robert 
Lahaise dir., Le Devoir, reflet du Québec au $X X^{c}$ siècle, Montréal, Hurtubise HMH, 1994, p. 99-103. Ce qui n'empêchera pas Ryan de voir son opinion triompher lorsqu'en 1982, la Cour suprême du Canada invalidera les articles de la charte traitant de la langue d'enseignement.

${ }^{73}$ Claude Ryan, «Pourquoi j'entre en politique, et au Parti libéral», dans Une société stable..., p. 51-58. 